\title{
Understanding the missing clusters problem at the Galactic center. Dissolved cluster(s) detection in the Sgr B1 region.
}

Francisco Nogueras-Lara ( $\square$ nogueras@mpia.de)

Max Planck Institute for Astronomy https://orcid.org/0000-0002-6379-7593

\section{Rainer Schödel}

Instituto de Astrofísica de Andalucía (CSIC) https://orcid.org/0000-0001-5404-797X

Nadine Neumayer

Max Planck Institute for Astronomy

\section{Article}

Keywords:

Posted Date: February 25th, 2022

DOI: https://doi.org/10.21203/rs.3.rs-1363061/v1

License: (c) (1) This work is licensed under a Creative Commons Attribution 4.0 International License.

Read Full License

Version of Record: A version of this preprint was published at Nature Astronomy on August 25th, 2022. See the published version at https://doi.org/10.1038/s41550-022-01755-3. 
Understanding the missing clusters problem at the Galactic center. Dissolved cluster(s) detection in the Sgr B1 region.

F. Nogueras-Lara ${ }^{1}$, R. Schödel ${ }^{2}$, and N. Neumayer ${ }^{1}$

${ }^{1}$ Max-Planck Institute for Astronomy, Königstuhl 17, 69117 Heidelberg, Germany

${ }^{2}$ Instituto de Astrofísica de Andalucía (CSIC), Glorieta de la Astronomía s/n, 18008 Granada, Spain

email:nogueras@mpia.de

The Milky Way's center is the closest galaxy nucleus and our Galaxy's most extreme environment ${ }^{1,2,3}$. Although its volume is less than $1 \%$ of the one of the Galactic disk, up to $10 \%$ of all new-born stars in the Galaxy in the past $100 \mathrm{Myr}$ formed there ${ }^{4,5,6,7}$. Therefore, it constitutes a perfect laboratory to understand star formation under extreme conditions ${ }^{3}$, similar to those in starburst or high-redshift galaxies. However, the only two known Galactic center young clusters account for $<10 \%$ of the expected young stellar mass ${ }^{8}$. Studying Sgr B1, a luminous Galactic center HII region ${ }^{9}$, we find evidence for the presence of several $10^{5} M_{\odot}$ of young stars, probably tracing one or several dissolved young clusters that formed $\sim 10 \mathrm{Myr}$ ago. This is a large step toward a better understanding of star formation at the Galactic center, such as the fate of young clusters, and the possibly different initial mass function in this region.

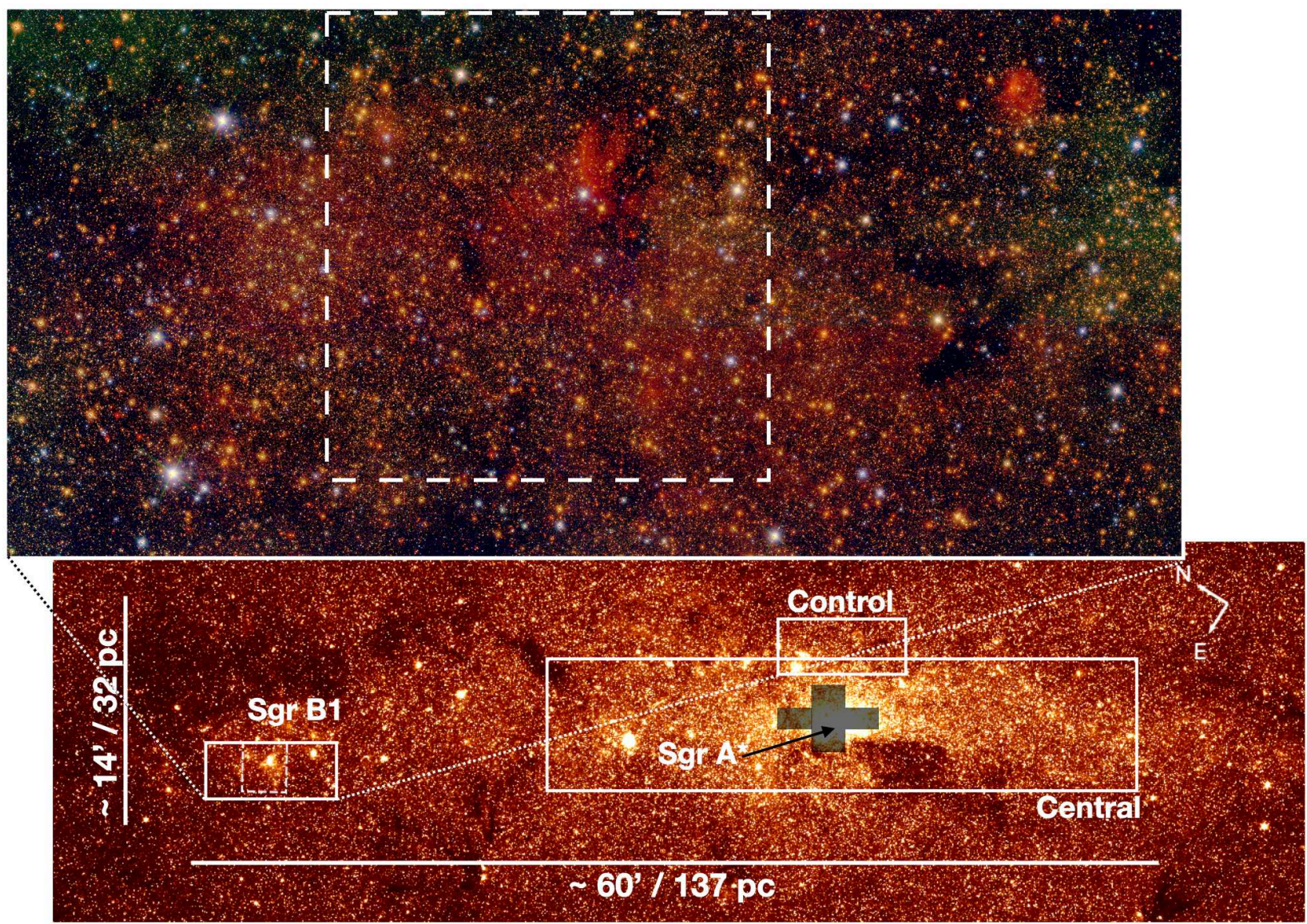

Fig. 1: Scheme of the analyzed regions (Sgr B1, control, and central nuclear disk) plotted over a Spitzer $4.5 \mu \mathrm{m}$ image ${ }^{28}$. The black shaded cross indicates a region dominated by the nuclear star cluster that was excluded from the analysis of the central field ${ }^{5}$. The Zoom-in region corresponds to a JHKs GALACTICNUCLEUS false color image. The dashed rectangle indicates the region of intense hot dust emission that was specifically studied. 
Studies of the radio to high energy emission, the finding of massive young stars throughout its region, the detection of classical cepheids, as well as the star formation history inferred from luminosity functions, all indicate that the star formation reached values of the order of $0.1 M_{\odot} / y r$, in the Galactic center in the past 10-100 $\mathrm{Myr}^{4,5,6,7,10}$. However, there are only two young massive clusters known (Arches and Quintuplet), that account for less than $10 \%$ of the total young stellar mass expected ${ }^{11}$. This is the so-called missing clusters problem. A plausible explanation for this is the rapid dissolution of even the most massive clusters, due to the tidal field in the Galactic center and encounters of the young clusters with massive molecular clouds ${ }^{8,12}$. Moreover, the high stellar background density ${ }^{13}$ combined with the strong and patchy interstellar extinction toward the Galactic center ${ }^{14}$ hamper the detection of all but the tightest and most massive young clusters as well as of individual young stars, making the creation of a complete census of recent star formation in this region a formidable challenge.

Sagittarius (Sgr) B1 is a well-known HII region in the Galactic center ${ }^{9}$. Far-infrared observations suggests that it contains substantial amounts of young massive stars ${ }^{9}$. Here we use the GALACTICNUCLEUS survey ${ }^{13,14}$ - a high-angular resolution ( $0.2^{\prime \prime}$ ) JHKs catalog specifically designed to observe the Galactic center ${ }^{13}$ - to study a field of $\sim 160 \mathrm{pc}^{2}$ covering part of the Sgr B1 region (centered on $17 \mathrm{~h} 47 \mathrm{~m} 15.41 \mathrm{~s}-28^{\circ} 31^{\prime} 39.7^{\prime}$, Fig. 1), and compare it with a control field of similar size in the inner Galactic center region (centered on $17 \mathrm{~h} 45 \mathrm{~m} 20.81 \mathrm{~s}-28^{\circ} 57^{\prime} 58.6^{\prime}$, Fig. 1). We chose this control field because it was observed under similar excellent conditions as the target field ${ }^{13}$ (seeing in $\mathrm{H}, \mathrm{Ks} \sim 0.4^{\prime \prime}$ ), and because it does not contain any obvious structures, such as the Arches or Quintuplet clusters or the nuclear star cluster.

Given the significantly different extinction along the line of sight between the Galactic center and the Galactic disk, we applied a color cut in the HKs color-magnitude-diagram to remove foreground stars $^{5,15}$ (Fig. 2a). We then built extinction maps using red giant stars - whose intrinsic color (H-Ks) is approximately constant - to correct for reddening and differential extinction ${ }^{16}$. Finally, we created a Ks luminosity function (KLF, Fig. 2b, corrected for completeness), that gives the number of stars per luminosity interval.

The KLF of a stellar population contains information about its formation history ${ }^{5}$. We fitted the KLFs of the Sgr B1 and the control fields with a linear combination of theoretical models applying Monte Carlo (MC) simulations (see Methods). The KLF of a single-age population changes as a function of age, with the variability time-scales shortening towards younger ages. We chose the age-sampling particularly closely spaced for the youngest ages (5-40 Myr). We used two different sets of stellar evolutionary models that properly cover the youngest stellar populations, to deal with possible systematics (Parsec ${ }^{17,18,19}$ and MIST $^{20,21,22}$ ). We assumed a metallicity of twice solar in agreement with recent results for the Galactic center ${ }^{5,23,24}$.

Figure 2 (c, d) shows the obtained star formation history (SFH) for the Sgr B1 and the control field. We also applied the same technique to a KLF of the central region of the nuclear stellar disk ${ }^{5}$ ( $1600 \mathrm{pc}^{2}$, indicated in Fig. 1), that does not contain the nuclear star cluster (whose SFH is significantly different ${ }^{5,25,26}$ ), to compare the SFHs (Fig. 2e). The results for the control and the inner Galactic center fields agree with previous work ${ }^{5}$ : The bulk of stars ( $\gtrsim 80 \%$ of the stellar mass) in both fields is older than $7 \mathrm{Gyr}$. There was a distinct epoch of star formation between 0.5-2 Gyr ago, where we found a significant contribution from the stellar models with ages of $\sim 1 \mathrm{Gyr}$, corresponding to the massive star forming event that took place $\sim 1 \mathrm{Gyr}$ ago in the nuclear stellar disk ${ }^{5}$. Star formation continued at lower level until the present moment. On the other hand, the SFH of the Sgr B1 region is significantly different. The stellar population is younger on average and there is an important contribution from an intermediate age (2-7 Gyr) population ( $40 \%$ of the total stellar mass), suggesting a more continuous $\mathrm{SFH}$ than in the innermost regions of the Galactic center. Moreover, the star formation activity between 0.5-2 Gyr is more prominent. Finally, we detect a much larger contribution from young stars. In particular, the youngest age bin ( $<60 \mathrm{Myr}$ ) accounts for more than $5 \%$ of the total stellar mass of the Sgr B1 region, six times more than in the control field.

The presence of a significantly different contribution from the intermediate age stellar population in the Sgr B1 region in comparison with the control and the inner Galactic center may be indicative of an inside-out formation of the nuclear stellar disk. Such an age gradient has also been 
observed in external galaxy nuclei and used to propose the inside-out formation channel of nuclear stellar disks ${ }^{27}$.

a

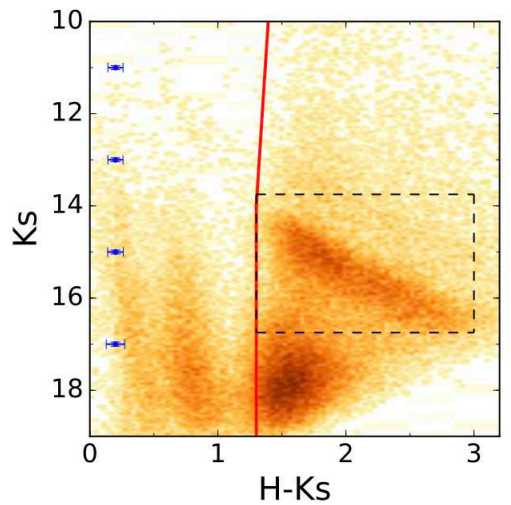

b

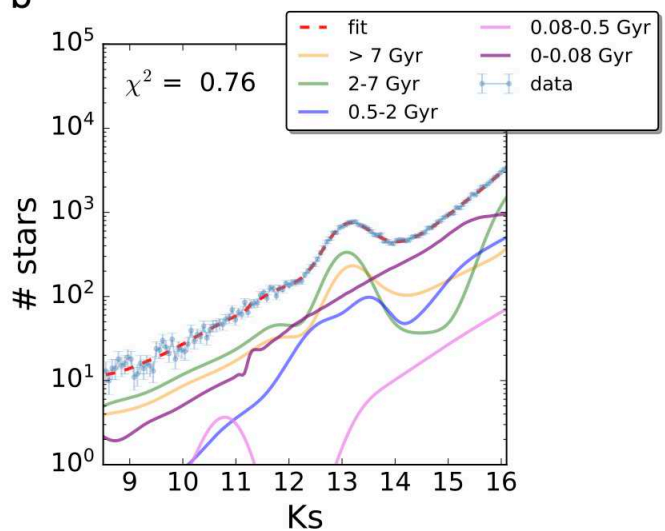

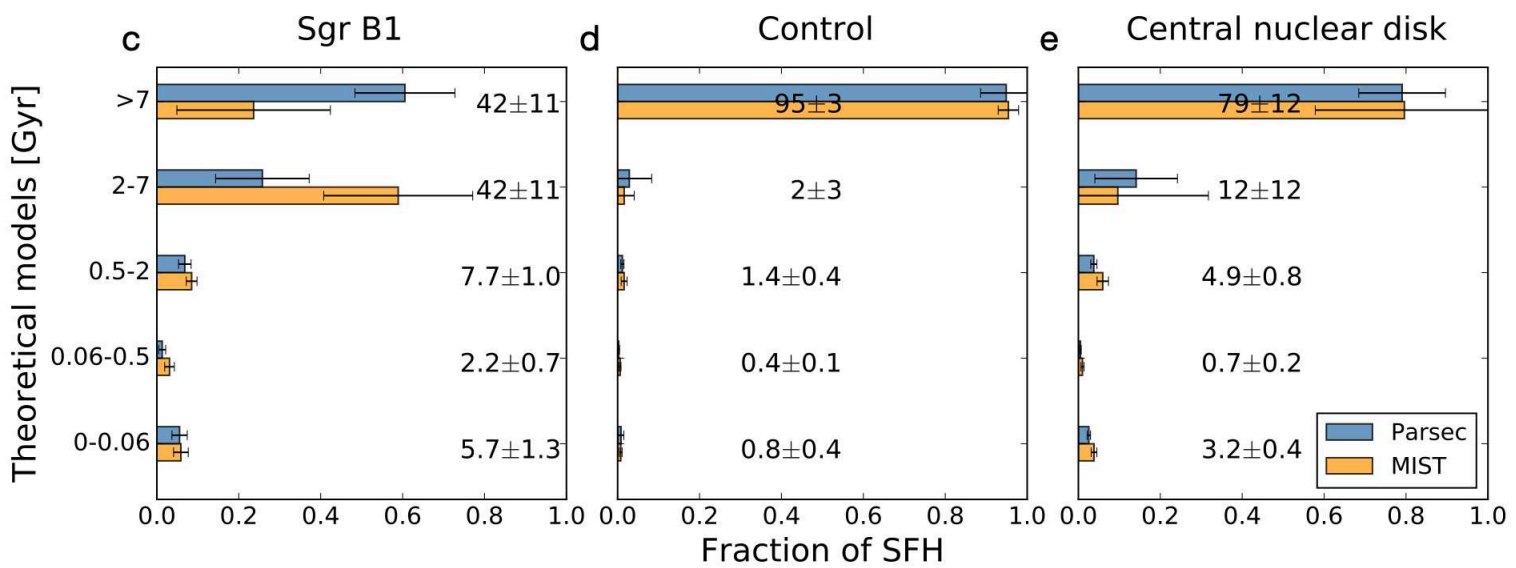

Fig. 2: a, H-Ks color-magnitude diagram (Vega magnitudes) for the Sgr B1 field. The red line shows the color cut to remove foreground stars. The black dashed rectangle indicates the red giant stars used to compute the extinction map. The blue error bars show the $1 \sigma$ mean uncertainties of the data. $\boldsymbol{b}, \mathrm{Sgr} B 1$ KLF (in blue) corrected for extinction, completeness, and saturation. The error bars show the $1 \sigma$ uncertainties per brightness bin. The red dashed line indicates the best-fit model (Parsec models). Colored lines depict the contribution of the different age bins. c, $d, e$, SFHs of the Sgr B1, the control, and the central region of the nuclear stellar disk. The error bars depict the $1 \sigma$ uncertainties. The numbers indicate the mean fractions of originally formed stellar mass and their uncertainties.

Using Parsec models, we estimate that the Sgr B1 field studied here contains a total originally created stellar mass of $(7.6 \pm 0.7) \cdot 10^{6} M_{\odot}$. This implies that the youngest stellar population accounts for $(4.3 \pm 1.1) \cdot 10^{5} M_{\odot}$, corresponding to around ten times the mass of the Arches and Quintuplet clusters, contained in a relatively small region of $\sim 160 \mathrm{pc}^{2}$. Given that there is not any clear stellar overdensity in this region, our results indicate the presence of one or several (partially) dissolved young cluster(s).

We further investigated the presence of young stars in Sgr B1 by carrying out a dedicated analysis of a small region ( $\sim 40 \mathrm{pc}^{2}$, white dashed rectangle in Fig. 1), that contains intense dust emission in the Spitzer image ${ }^{28}(4.5 \mu \mathrm{m})$, probably caused by young hot stars. We found that more than $7 \%$ of the total stellar mass is due to young stars ( $<60 \mathrm{Myr})$. We also estimated the age of the stars in this region by analyzing the contribution of the considered young stellar models $(5,10,20$, and $40 \mathrm{Myr}$ ) to each of the MC samples (see Methods). Using Parsec and MIST models, we found that the 5 and 10 Myr model populations contribute significantly to $99 \%$ of all MC samples, and correspond to $~ 6 \%$ of the total stellar mass (Fig. 3). Therefore, we estimate that this region contains $\sim 1.2 \cdot 10^{5} M_{\odot}$ of stars with ages between 5-10 Myr. A similar analysis carried out for the whole Sgr B1 field indicates that 
$\sim 2 \%$ of the total stellar mass is due to young stars with ages between 5-10 Myr. We thus conclude that the $\sim 40 \mathrm{pc}^{2}$ region with intense hot dust emission presents an overabundance of young stars in comparison with the surrounding area.

Our results are consistent with the presence of several massive stars in the Sgr B1 region ${ }^{10}$ that might be associated with the potential dissolved cluster(s). In particular the presence of 3 Wolf Rayet stars can be interpreted as an age estimator of the detected dissolved cluster(s) that agree with the estimated age of between 5 and 10 Myr. Furthermore, recent KMOS observations have detected evidence of an apparent over density of mid-O super-/hypergiants and WNLha-type stars spatially coincident with the Sgr B1 complex ${ }^{29}$. On the other hand, SOFIA FIFI-LS observations have shown that the ionized gas in the Sgr B1 region must be due to stars widely spread throughout the field, and pointed towards the presence of a young stellar population that were not formed in situ?

Our detection of a (several) massive young dissolved cluster(s) in the Sgr B1 region is fundamental to shed new light on star formation in the Galactic center. Our findings agree with a rapid dispersion of young clusters below the background density in the Galactic center. In this way, the age that we estimate for the dissolved cluster(s) is consistent with the predicted very short disruption time of 6 Myr caused by tidal shocking by giant molecular clouds in the Galactic center ${ }^{12}$. Moreover, it also agrees with the previously inferred recent star formation history in the Galactic center that presents a maximum of star formation around $10 \mathrm{Myr} \mathrm{ago}^{30}$, that is compatible with the age of the detected dissolved cluster(s).

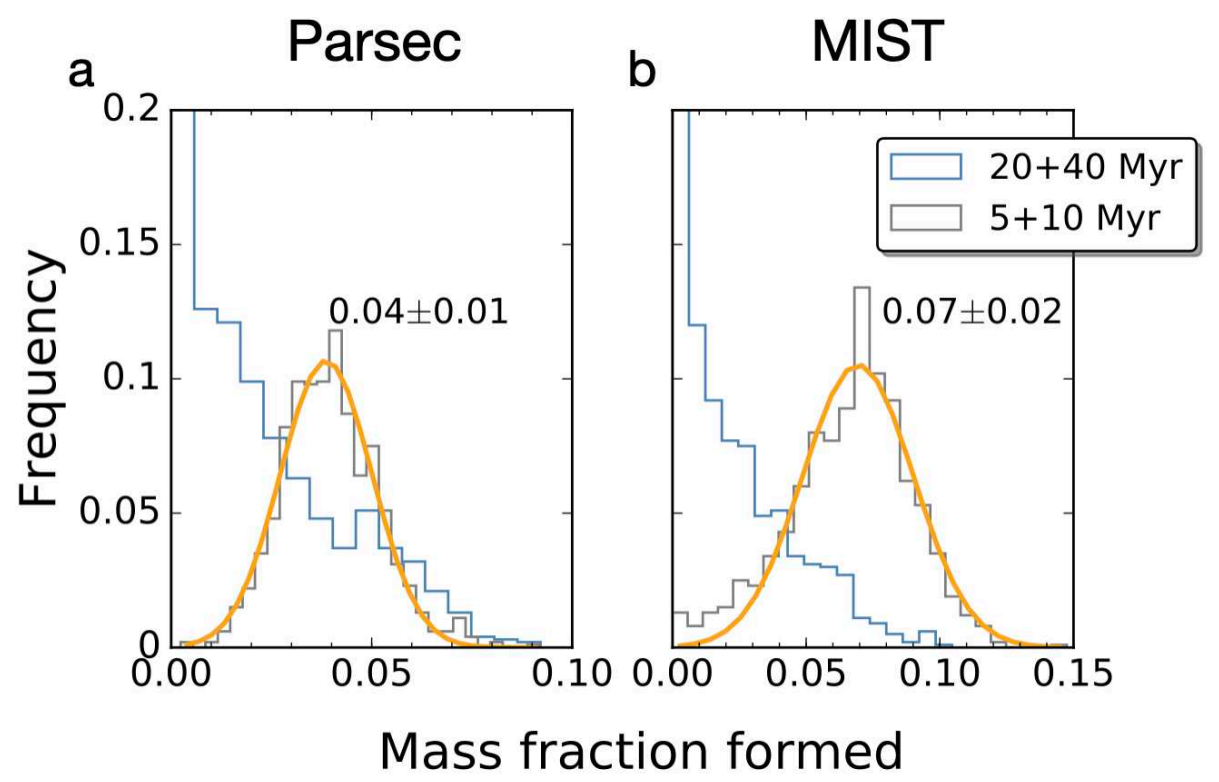

Fig. 3: Young models' contribution to the KLF of the Sgr B1 hot dust emission region using Parsec (a) and MIST (b) models. The yellow lines show a Gaussian fit to the distribution of $5+10$ Myr. The mean and the standard deviation (1 $\sigma$ uncertainty) are indicated in each panel.

To our knowledge, our results constitute the first detection of a significant mass of recently formed stars at the Galactic center beyond the Arches and Quintuplet clusters and the known isolated massive stars. Our findings may indicate the fate of the Arches and Quintuplet clusters, that are around 5 million years younger than the detected young stellar population. They also help understand the isolated massive stars detected across the Galactic center, whose proper motions indicate they are not related to the so-far known young clusters ${ }^{31}$, supporting their formation in clusters that have dispersed on rather short timescales after their formation several million years ago. 


\section{Data Availability}

All the raw data used in this study are available at the ESO Science Archive Facility (http://archive.eso.org/eso/eso archive main.html) under program ID 091.B-0418. The final version of the GALACTICNUCLEUS survey (images and point source catalogues) is publicly available via the ESO phase 3 data release interface (https://www.eso.org/qi/catalogQuery/index/369).

\section{Author contributions}

F. N.-L. reduced the data, carried out the analysis, and wrote the manuscript. R. S. produced the MIST KLFs, and N. N. and R.S. contributed to the interpretation and the discussion of the results, and helped organizing and editing the manuscript in its final version.

\section{Acknowledgements}

This work is based on observations made with ESO Telescopes at the La Silla Paranal Observatory under program ID 091.B-0418. We thank the staff of ESO for their great efforts and helpfulness. F.N.-L. acknowledges the sponsorship provided by the Federal Ministry for Education and Research of Germany through the Alexander von Humboldt Foundation. F.N.-L. and N.N. gratefully acknowledge support by the Deutsche Forschungsgemeinschaft (DFG, German Research Foundation) Project-ID 138713538 SFB 881 ("The Milky Way System", subproject B8). R.S. acknowledges financial support from the State Agency for Research of the Spanish MCIU through the "Center of Excellence Severo Ochoa" award for the Instituto de Astrofísica de Andalucía (SEV-2017-0709). R.S. acknowledges financial support from national project PGC2018-095049-B-C21 (MCIU/AEI/FEDER, UE).

[1] Nishiyama, S., Nagata, T., Tamura, M., Kandori, R., Hatano, H., Sato, S. \& Sugitani, K. The Interstellar Extinction Law toward the Galactic Center. II Y, J, H, and Ks Bnads. Astrophys. J. 680, 1174-1179 (2008). [2] Launhardt, R., Zylka, R. \& Mezger, P. G. The nuclear bulge of the Galaxy. III. Large-scale physical characteristics of stars and interstellar matter. Astron. Astrophys. 384, 112-139 (2002).

[3] Morris, M. \& Serabyn, E. The Galactic Center Environment. Annu. Rev. Astron. Astrophys. 34, 645701 (1996).

[4] Matsunaga, N. Kawadu, T., Nishiyama, S., Nagayama, T., Kobayashi, N. Tamura, M., Bono, G., Feast, M. W. \& Nagata, T. Three classical Cepheid variable stars in the nuclear bulge of the Milky Way. Nature 477, 188-190 (2011).

[5] Nogueras-Lara, F., Schödel, R., Gallego-Calvente, A. T., Gallego-Cano, E., Banafsheh, S., Dong, H., Neumayer, N., Hilker, M., Najarro, F., Nishiyama, S., Feldmeier-Krause, A. \& Girard, J. H. V. Early formation and recent starburst activity in the nuclear disk of the Milky Way. Nature Astronomy 4, 377381 (2020).

[6] Barnes, A. T., Longmore, S. N., Battersby C., Bally, J., Kruijssen, J. M. D., Henshaw, J. D., \& Walker, D. L., Star formation rates and efficiencies in the Galactic Centre, MNRAS, 469, 2263-2285 (2017).

[7] Crocker, R. M., Jones, D. I., Aharonian, F., Law, C. J., Melia, F., Oka, T., \& Ott, J., Wild at Heart: the particle astrophysics of the Galactic Centre, MNRAS, 431, 763-788 (2011).

[8] Portegies-Zwart, S. F., Makino, J., McMillan, S. L. W. \& Hut, P. The Lives and Deaths of Star Clusters near the Galactic Center. Astrophys. J. 565, 265-279. (2010).

[9] Simpson, J. P., Colgan, S. W. J., Cotera, A. S., Kaufman, M. J. \& Stolovy, S. R. SOFIA FIFI-LS Observations of Sgr B1: Ionization Structure and Sources of Excitation. ApJL. 867: L13 (2018).

[10] Mauerhan, J. C., Muno, M. P., Morris, M. R., Stolovy, S. R., \& Cotera, A. Near-infrared Counterparts to Chandra X-ray Sources Toward the Galactic Center. II. Discovery of Wolf-Rayet Stars and O Supergiants. ApJ. 710, 706 (2010). 
[11] Schneider, F. R. N., Izzard, R. G., de Mink, S. E., Langer, N., Stolte, A., de Koter, A., Gvaramadze, V. V., Hußmann, B., Liermann, A. \& Sana, H. Ages of Young Star Clusters, Massive Blue Stragglers, and the Upper Mass Limit of Stars: Analyzing Age-dependent Stellar Mass Functions. ApJ. 780: 117 (2014).

[12] Kruijssen, J. M. D., Longmore, S. N., Elmegreen, B. G., Murray, N. Bally, J., Testi, L. \& Kennicut, R. C. What controls star formation in the central 500 pc of the Galaxy? MNRAS, 440, 3370-3391 (2014).

[13] Nogueras-Lara, F., Schödel, R., Gallego-Calvente, A. T., Dong, H., Gallego-Cano, E., Shahzamanian, B., Girard, J. H. V., Nishiyama, S., Najarro, F. \& Neumayer, N. GALACTICNUCLEUS: A high angular resolution JHKs imaging survey of the Galactic centre. II. First data release of the catalogue and the most detailed CMDs of the GC. Astron. Astrophys. 631, A20 (2019).

[14] Nogueras-Lara, F., Gallego-Calvente, A. T., Dong, H., Gallego-Cano, E., Girard, J. H. V., Hilker, M., de Zeeuw, P. T., Feldmeier-Krause, A., Nishiyama, S., Najarro, F., Neumayer, N. \& Schödel, R. GALACTICNUCLEUS: A high angular resolution JHKs imaging survey of the Galactic centre. I. Methodology, performance, and near-infrared extinction towards the Galactic centre. Astron. Astrophys. 610, A83 (2018).

[15] Nogueras-Lara, F., Schödel, R. \& Neumayer, N. Distance and extinction to the Milky Way spiral arms along the Galactic centre line of sight. Astron. Astrophys. 653, A33 (2021).

[16] Nogueras-Lara, F., Schödel, R. \& Neumayer, N. GALACTICNUCLEUS: A high-angular-resolution JHKs imaging survey of the Galactic centre. IV. Extinction maps and de-reddened photometry. Astron. Astrophys. 653, A133 (2021).

[17] Bressan, A., Marigo, P., Girardi, L., Salasnich, B., Dal Cero, C. \& Ambra N. PARSEC: stellar tracks and isochrones with the PAdova and TRieste Stellar Evolution Code. MNRAS, 427, 127 (2012).

[18] Chen, Y., Girardi, L., Bressan, A., Marigo, P., Barbieri, M. \& Xu, K. Improving PARSEC models for very low mass stars. MNRAS, 444, 2525 (2014).

[19] Chen, Y., Bressan, A., Girardi, L., Marigo, P., Xu, K \& Lanza, A. PARSEC evolutionary tracks of massive stars up to $350 \mathrm{M}_{\odot}$ at metallicities $0.0001<Z<0.04$. MNRAS, 452, 1068-1080 (2015).

[20] Dotter, A. MESA Isochrones and Stellar Tracks (MIST) 0: Methods for the Construction of Stellar Isochrones. APJS 222, 8 (2016).

[21] Choi, J., Dotter, A., Conroy, C., Cantiello, M., Paxton, B. \& Johnson, B. D. Mesa Isochrones and Stellar Tracks (MIST). I. Solar-scaled Models. ApJ 823, 102 (2016).

[22] Paxton, B., Bildsten, L., Dotter, A., Herwig, F., Lesaffre, P. \& Timmes, F. Modules for Experiments in Stellar Astrophysics (MESA). APJS 192, 3 (2011).

[23] Schultheis, M., Rich, R. M., Origlia, L., Ryde, N., Nandakumar, G., Thorsbro, B. \& Neumayer, N. The inner two degrees of the Milky Way. Evidence of a chemical difference between the Galactic Center and the surrounding inner bulge stellar populations. Astron. Astrophys. 627, A152 (2019).

[24] Schultheis, M., Fritz, T. K., Nandakumar, G., Rojas-Arriagada, A., Nogueras-Lara, F., FeldmeierKrause, A., Gerhard, O., Neumayer, N., Patrick, L. R., Prieto, M. A., Schödel, R., Mastrobuono-Battisti, A. \& Sormani, M. C. The nuclear stellar disc of the Milky Way: A dynamically cool and metal-rich component possibly formed from the central molecular zone. Astron. Astrophys. 650, A191 (2021).

[25] Schödel, R., Nogueras-Lara, F., Gallego-Cano, E., Shahzamanian, B., Gallego-Calvente, A. T. \& Gardini, A. The Milky Way's nuclear star cluster: Old, metal-rich, and cuspy. Structure and star formation history from deep imaging. Astron. Astrophys. 641, A102 (2020).

[26] Nogueras-Lara, F., Schödel, R. \& Neumayer, N. The Nuclear Star Cluster and Nuclear Stellar Disk of the Milky Way: Different Stellar Populations and Star Formation Histories. ApJ. 920: 97 (2021).

[27] Bittner, A., Sánchez-Blázquez, P., Gadotti, D. A., Neumann, J., Fragkoudi, F., Coelho, P., de LorenzoCáceres, A. Falcón-Barroso, J., Kim, T. Leaman, R., Martín-Navarro, I., Méndez-Abreu, J., Pérez, I., Querejeta, M., Seidel, M. K. \& van de Ven, G. Inside-out formation of nuclear discs and the absence of old central spheroids in barred galaxies of the TIMER survey. Astron. Astrophys. 643, A65 (2020).

[28] Stolovy, S., Ramirez, S., Arendt, R. G., Cotera, A., Yusef-Zadeh, F., Law, C., Gezari, D., Sellgren, K., Karr, J., Moseley, H., Smith, H. A. A mid-infrared survey of the inner 2x1.5 degrees of the Galaxy with Spitzer/IRAC. J. Phys. Conf. Ser., 54, 176 (2006).

[29] Clark, J. S., Patrick, L. R., Najarro, F., Evans, C. J., Lohr, M. Constraining the population of isolated massive stars within the Central Molecular Zone. Astron. Astrophys. 649, A43 (2021). 
[30] Armillotta, L., Krumholz, M. R., Di Teodoro, E. M. \& McClure-Griffiths, N. M. The life cycle of the Central Molecular Zone - I. Inflow, star formation, and winds. MNRAS, 490, 4401-4418 (2019).

[31] Libralato, M., Lennon, D. J., Bellini, A., van der Marel, R., Clark, S. J., Najarro, F., Patrick, L. R, Anderson, J. Bedin, L. R., Crowther, P. A., de Mink, S. E., Evans, C. J., Platais, I., Sabbi, E., Sohn \& S. T. 2D kinematics of massive stars near the Galactic Centre. MNRAS, 500, 3213-3239 (2021).

[32] Nishiyama, S., Nagata, T., Kusakabe, N., Matsunaga, N., Naoi, T. et al. Interstellar Extinction Law in the J, H, and Ks Bands toward the Galactic Center. Astrophys. J. 638, 839-846 (2006).

[33] Girardi, L. Red Clump Stars, Annu. Rev. Astron. Astrophys. 54, 95-133 (2016).

[34] Nogueras-Lara, F., Schödel, R., Neumayer, N., Gallego-Cano, E., Shahzamanian, B., GallegoCalvente, A. T. \& Najarro, F. GALACTICNUCLEUS: A high-angular-resolution JHKs imaging survey of the Galactic centre. III. Evidence for wavelength-dependence of the extinction curve in the near-infrared. Astron. Astrophys. 641, A141 (2021).

[35] Freedman, D. \& Diaconis, P. On the histogram as a density estimator: L2 theory. Probability Theory and Related Fields, 57, 453 (1981).

[36] Sturges, H. A. The Choice of a Class Interval. Journal of the American Statistical Association, 21, 153 (1926).

[37] Diolaiti, E., Bendinelli, O., Bonaccini, D., Close, L.-M., Currie, D.-G. \& Parmeggiani, G. StarFinder: an IDL GUI-based code to analyze crowded fields with isoplanatic correcting PSF fitting. Society of Photo-Optical Instrumentation Engineers (SPIE) Conference Series, 4007, 879 (2000).

[38] Matsunaga, N., Kawadu, T., Nishiyama, S., Nagayama, T., Hatano, H., Tamura, M., Glass, I.-S. \& Nagata, T. A near-infrared survey of Miras and the distance to the Galactic Centre. MNRAS, 399, 17091729 (2009).

[39] Eisenhauer, F., Quirrenbach, A., Zinnecker, H., \& Genzel, R. Stellar Content of the Galactic Starburst Template NGC 3603 from Adaptive Optics Observations. ApJ, 498, 278 (1998).

[40] Rui, N. Z., Hosek, M. W. Jr., Lu, J., Clarkson, W. I., Jay, A., Morris, M. A. \& Ghez, A. The Quintuplet Cluster: Extended Structure and Tidal Radius. ApJ 877, 37 (2019).

[41] Najarro, F., Figer, D.-F., Hillier, D.-J., Geballe, T.-R. \& Kudritzki, R.-P. Metallicity in the Galactic Center: The Quintuplet Cluster. ApJ 691, 1816 (2009).

\section{Methods}

\section{Data}

For this work we used $\mathrm{H}$ and $\mathrm{Ks}$ data from the GALACTICNUCLEUS survey ${ }^{13,14}$, that are publicly available on the ESO Phase 3 data release archive. This is a high-angular resolution ( $\left.0.2^{\prime \prime}\right)$ JHKs survey of the Galactic center especially designed to observe its stellar population overcoming the extreme extinction and source crowding. It contains accurate photometry of $\sim 3.3 \cdot 10^{6}$ stars covering a total area of $\sim 6000 \mathrm{pc}^{2}$. The photometric statistical uncertainties are below 0.05 mag at $\mathrm{H}^{\sim 19}$ and $\mathrm{Ks} \sim 18$ mag. The zero-point systematic uncertainty is $\$ 0.04$ mag in all bands. In particular, we used two individual pointings (D12 and F19) ${ }^{13}$, partially covering the Sgr B1 region and a control field, that were observed under similar excellent conditions (seeing in $\mathrm{H}, \mathrm{Ks} \sim 0.4^{\prime \prime}$ ). As comparing fields, we also used data from pointing F10, containing the Quintuplet cluster, and the 14 central pointings of the survey that cover the central region of the nuclear stellar disk ${ }^{5}$. For the latter case, we directly used the final KLF as it was obtained in previous work ${ }^{5}$.

Prior to our analysis, we corrected potential saturation problems in Ks for stars brighter than $11.5 \mathrm{mag}^{13}$. For this, we used the SIRIUS IRSF ${ }^{32}$ survey of the Galactic center to replace the photometry of saturated stars, and also completed the list with bright stars that might have escaped detection in the GALACTICNUCLEUS catalogue.

\section{Extinction maps}

For each of the analyzed regions, we created a dedicated extinction map using red clump stars $^{33}$ (red giant stars in their helium burning phase), that are very abundant and homogeneously 
distributed across the field, and have a well-defined intrinsic color ${ }^{16}(\mathrm{H}-\mathrm{Ks})_{0}=0.10 \pm 0.01 \mathrm{mag}$. We also included red giant stars (with very similar intrinsic colors $\mathrm{H}-\mathrm{Ks})^{5,16}$, to increase the angular resolution of the maps. To choose the reference stars, we used a color cut in the color-magnitude diagrams as shown in Fig. 2a (black dashed rectangle). We built the extinction maps following the methodology described in our previous work ${ }^{16}$ and assuming an extinction curve ${ }^{34} A_{H} / A_{k s}=1.84 \pm 0.03$. We defined a pixel size of 2", and computed the associated extinction values for each pixel by using the five closest reference stars in a maximum radius of $7.5^{\prime \prime}$. We weighted the distances using an inverse-distance weight method, and assumed a maximum color difference of 0.3 mag between the stars to avoid mixing stars with too different extinction. If less than five reference stars were detected for a given pixel, we did not assign any extinction value ("NaN"). Figure 4 shows the extinction maps obtained for the Sgr B1 and the control field (F19). Using a Jackknife resampling method, systematically leaving out one of the reference stars for each pixel, we obtained a statistical uncertainty of the extinction maps of $\sim 3 \%$. The systematics were estimated quadratically propagating the uncertainties of the quantities involved in the extinction calculation. We obtained a mean systematic uncertainty of $\sim 5 \%$.
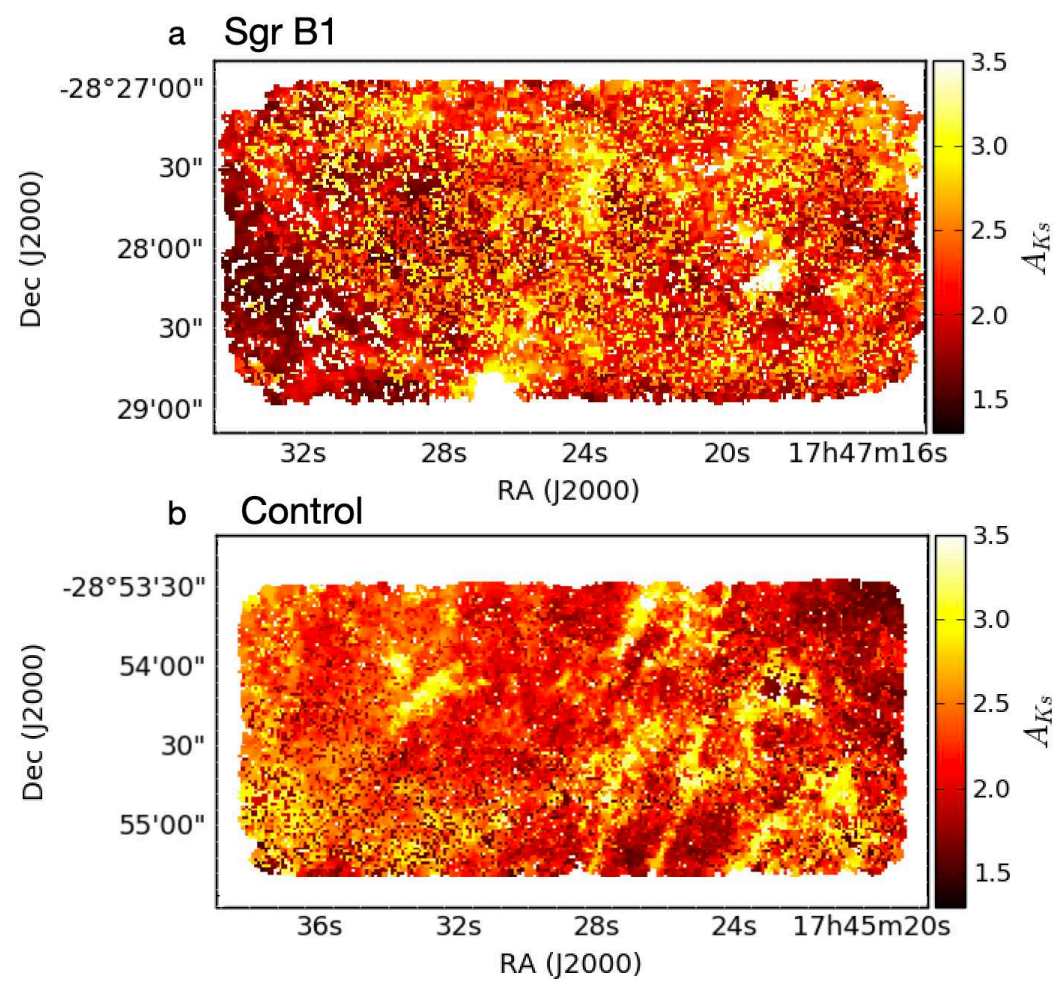

Fig. 4: Extinction maps for Sgr B1 (a) and the control field (b). White pixels indicate "NaN" values, meaning that there were not enough reference stars to compute an associated extinction value.

\section{De-reddening}

Given the extreme differential extinction along the observed line of sight, it is possible to remove the foreground stellar population - belonging to the Galactic disk and bulge- by applying a color cut $\mathrm{H}-\mathrm{Ks}^{\sim} 1.3 \mathrm{mag}^{15}$ ( $\mathrm{red}$ solid line in Fig. 2a). We then applied the previously computed extinction maps to deredden each of the studied fields. Figure 5 shows the color-magnitude diagrams $\mathrm{H}-\mathrm{Ks}$ of the Sgr B1 field and the control region before and after applying the extinction maps. To check that the differential extinction is significantly corrected, we computed the standard deviation of the distribution of red clump stars before and after the extinction correction, and obtained that the scatter of red clump stars is $\sim 8$ times lower in the corrected sample. 


\section{a Sgr B1}

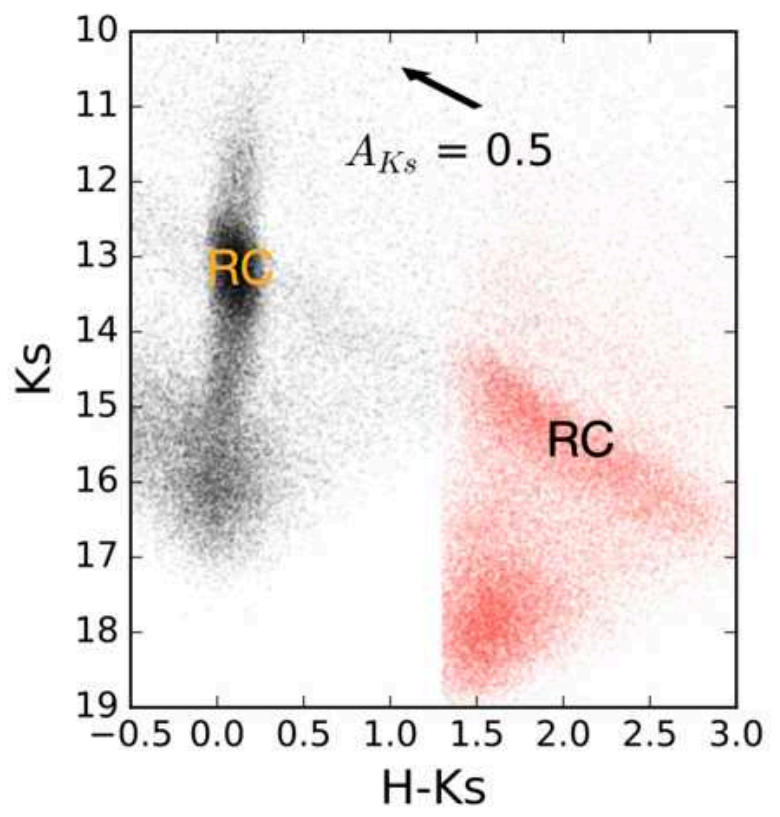

b Control

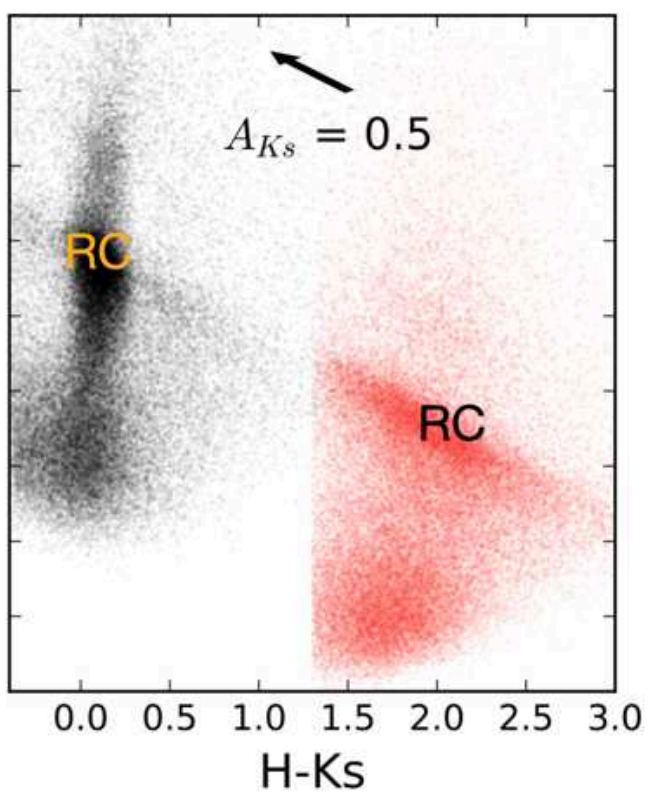

Fig. 5: Color-magnitude diagrams Ks versus H-Ks (Vega magnitude system) before (red dots) and after applying the extinction maps (black dots) for the Sgr B1 (a) field and the control region (b). The black arrows in the panels indicate the reddening vector for an extinction in $\mathrm{Ks}$ of $0.5 \mathrm{mag}$. The position of the red clump $(R C)$ is indicated before and after the correction.

\section{Ks Luminosity Function}

To create the KLFs, we used all the Ks de-reddened stars after excluding the foreground population ${ }^{5}$. We also removed over-de-reddened stars by excluding stars that are more than $2 \sigma$ brighter than the mean distribution of the de-reddened red clump feature. We created the KLF

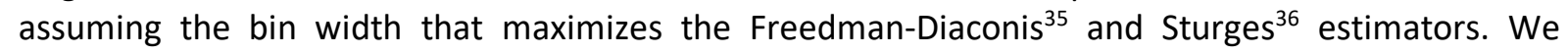
computed the uncertainties considering Poisson errors (i.e. the square root of the number of stars per bin).

\section{Completeness}

We also corrected for completeness computing a solution based on artificial stars tests. Namely, we created 20 modified science images for each field inserting $\sim 5 \%$ of the total number of stars, in magnitude bins of 0.5 mag starting from Ks=12 mag. We then used the StarFinder software ${ }^{37}$ to compute point spread function photometry following the same procedure used to create the GALACTICNUCLEUS survey ${ }^{13,14}$, and checked the fraction of recovered artificial stars to estimate the completeness correction. Figure 6 indicates the completeness solution for the Sgr B1 and the control fields. The uncertainties were estimated via the standard deviation of the completeness solution of the results obtained for each of the four independent HAWK-I chips that constitute each field ${ }^{14}$. We found that the completeness is higher for the Sgr B1 region, what can be explained due to the higher stellar crowding in the control field that is closer to the innermost regions of the Galactic center.

We computed an extinction correction for each completeness solution by calculating the median extinction of the stars constituting each KLF. We then completed the KLFs setting a lower limit of $75 \%$ of data completeness. We estimated the uncertainty per magnitude bin quadratically propagating the uncertainties from the completeness solution and the original $\mathrm{KLF}^{5}$. 


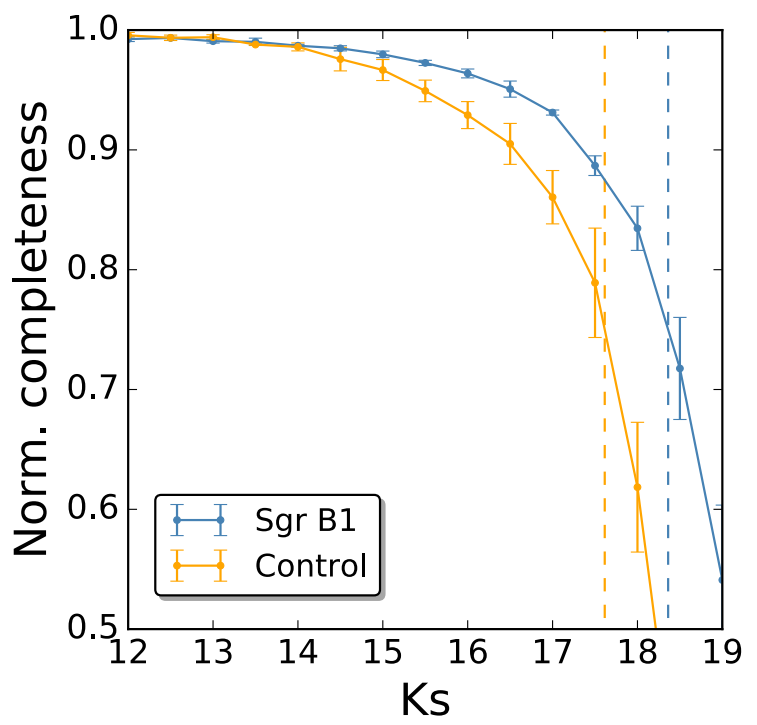

Fig. 6: Completeness solution for the Sgr B1 region and the control field. The $1 \sigma$ uncertainty is indicated in each case. The vertical dashed lines show the $75 \%$ level of completeness for each region.

\section{Saturation}

We restricted the analysis of the KLF to Ks>8.5 mag to avoid problems due to potential saturation of bright stars in the SIRIUS IRSF catalogues ${ }^{38}$.

\section{Model fitting and SFH calculation}

The KLF contains fundamental information about the SFH that can be reconstructed studying the characteristics of its main features ${ }^{5,25,26}$ (giant branch bump, red clump bump, and red giant branch bump), and their relative contribution to the total KLF. In this way, we checked that the KLFs from the Sgr B1 and the control regions are significantly different, probably indicating the presence of different stellar populations as we later confirmed (Fig. 7).

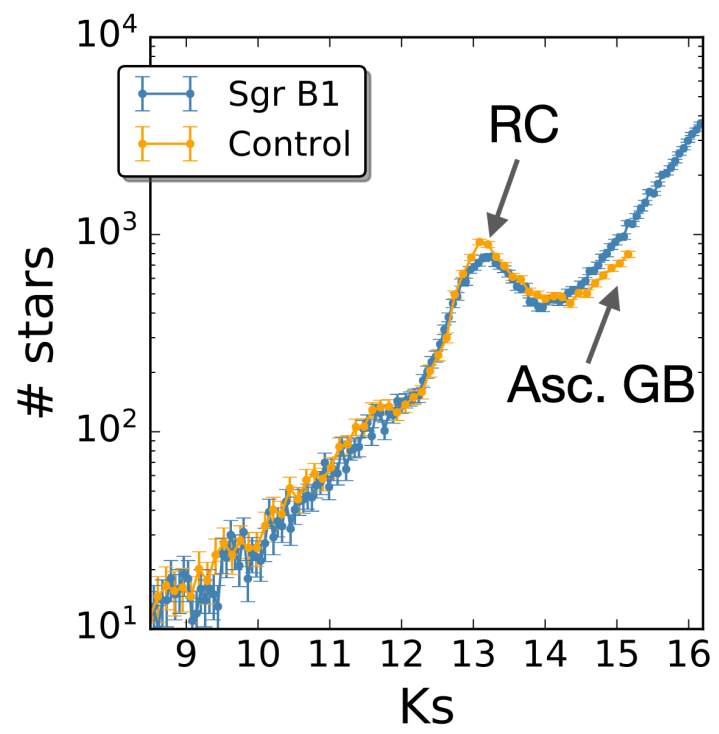

Fig. 7: KLFs comparison between the Sgr B1 region and the control field. The control region KLF was scaled to the Sgr B1's one, for an easier comparison, given their different stellar masses and bin widths. The error bars correspond to $1 \sigma$ uncertainties. The position of the red clump feature (RC) and the ascending giant branch, that present significant deviation between the KLFs, are shown in the figure. 
We derived the SFH of each of the studied regions by fitting the KLFs with a linear combination of theoretical models ${ }^{5}$. To search for young stars that are potential tracers of dissolved clusters, we used PARSEC models ${ }^{17,18,19}$ as a reference, given that they properly cover young stellar ages. We chose 14 individual ages for the model fit, that homogeneously sample the possible ages of the analyzed stellar populations: $14,11,8,6,3,1.5,0.6,0.4,0.2,0.1,0.04,0.02,0.01,0.005$ Gyr. We assumed a Kroupa initial mass function (IMF), and a stellar metallicity of around twice solar $(Z=0.03)$, in agreement with previous results for the Galactic center. To fit the KLFs, we included a parameter to account for the distance modulus ( 14.6 at the Galactic center distance), and allowed it to varied within $3 \sigma$ of the quadratically propagated uncertainties of the distance and the uncertainty of the dereddening process. We also included a Gaussian smoothing parameter to account for possible distance and/or differential extinction variations of the considered stellar populations.

To compute the SFH of each region, we resorted to Monte Carlo (MC) simulations creating $1000 \mathrm{KLFs}$ obtained by randomly varying the number of stars per bin assuming the $1 \sigma$ uncertainty as the standard deviation of the distribution. We then fitted each of the MC samples using a chi2 minimization criterion and obtained the SFH as the average of the results. To minimize possible degeneracies between models with similar age, we combined them into 5 final age-bins, as shown in Fig. 2 c, d, e. The $1 \sigma$ uncertainty is obtained via the standard deviation of the results in each age bin. To address potential systematic effects due to model selection, we repeated the procedure assuming MIST models ${ }^{20,21,22}$, that are independently created and also adequate to trace the young stellar ages. We chose similar ages, a metallicity of around twice solar, and a Salpeter IMF. The final value for each of the 5 age bins was computed averaging over the results obtained with Parsec and MIST. We estimated the final uncertainties quadratically propagating the ones independently obtained by using Parsec and MIST models.

We fitted the KLFs computed for the Sgr B1 and the control regions, and also the KLF derived for the central region of the nuclear stellar disk in our previous work ${ }^{5}$. We found a significantly different $\mathrm{SFH}$ in the Sgr B1 region, where there is a decrease of the contribution of old stars compensated by an increase of intermediate age stars, and a significant contribution of young stars, suggesting the presence of one (several) dissolved cluster(s).

We analyzed potential sources of systematic uncertainties to assess the obtained results:

(1) Stellar metallicity. We used Parsec models assuming solar and 1.5 solar metallicity to assess the results in the Sgr B1 region. The results agree within the uncertainties with the obtained for twice solar metallicity using Parsec models. The only difference is a somewhat higher contribution of the youngest stellar population for lower metallicities ( $\sim \%$ and $\sim 8 \%$ for 1.5 solar and solar metallicity, respectively).

(2) KLFs bin width. We analyzed the possible influence of the bin width on the derived SFH by repeating the process for new Sgr B1 KLFs assuming half and double the previously computed bin width $(0.03$, and $0.12 \mathrm{mag}$, respectively). The results are consistent with those obtained using Parsec models and a bin width of 0.06 mag.

(3) Faint end of the KLF. Given the different completeness of the Sgr B1 and the control fields (Sect. IV), we studied the influence of the deeper Sgr B1 photometry on the results. In this way, we repeated the analysis restricting the Sgr B1 KLF to the faint-end limit of the KLF of the control region (15.36 mag). We did not observe any significant difference within the uncertainties.

(4) Bright end of the KLF. We restricted the study of the KLFs to a Ks $>8.5$ mag limit to avoid any saturation problems related to the SIRIUS IRSF survey. Nevertheless, we also repeated the analysis of the Sgr B1 KLF considering a less conservative limit of Ks>7.5 mag. We concluded that there is not any significant variation on the results within the uncertainties. 
(5) Completeness solution. We based our completeness corrections on artificial stars tests. To assess our completeness solution, we also estimated the completeness using an alternative approach based on the determination of the critical distance at which a star of a given magnitude can be detected around a brighter star ${ }^{39}$. This method is less accurate and constitutes a rough completeness estimation that mainly accounts for the completeness due to crowding. We obtained that the completeness is $\sim 5-10 \%$ lower than in the case of using the artificial stars test. We checked whether this different completeness solution influences our results, repeating the Sgr B1 KLF analysis by assuming the new completeness solution. We did not observe any variation within the uncertainties.

(6) Different IMFs. There is some evidence of a top-heavy IMF for the known young clusters at the Galactic center ${ }^{40}$. In this way, we repeated the analysis of the Sgr B1 KLF considering an IMF with $\alpha=1.8$ using MIST models and twice the solar metallicity. We found that the SFH significantly changed for old ages, being the contribution from stellar models $>7 \mathrm{Gyr}$ shifted toward the 0.5-2 Gyr age bin. Nevertheless, the results for the youngest stellar bin did not change within the uncertainties, remaining the conclusion about the young stars unaffected. We believe that the age shift for the oldest stellar population is due to a probably necessary consideration of a stellar population enhanced in alpha elements ${ }^{41}$, when assuming a topheavy IMF, that was not considered due to the limitations of current models. Moreover, this alpha enhancement is required only for the young stars and might not be representative of the bulk of stars, making it probably necessary to use different metallicities and enhancement in alpha elements for stellar populations with different ages. On the other hand, we obtained that the mean chi2 computed for the described top-heavy models is significantly higher than for the standard case of using twice solar metallicity without enhancement in alpha elements. In any case, we conclude that the results for the youngest stellar population are robust.

\section{Total mass estimation}

We estimated the total stellar mass in the Sgr B1 region using the results obtained by fitting Parsec models. For this, we scaled the bin width of the KLF to the theoretical models' one and computed the stellar mass for each of the MC samples by combining the contributions of all the models in a given fit. The final stellar mass was obtained averaging over the results for each of the MC samples, where the associated uncertainty is the standard deviation of the mass distribution.

\section{Region of intense hot dust emission}

We carried out a dedicated analysis of the central region of Sgr B1, where the presence of hot dust emission is more intense than in the surrounding area based on a Spitzer $4.5 \mu \mathrm{m}$ image (Fig. 1). We created a KLF and fitted it following the previously explained procedure. Figure 8 shows the obtained results. We found that the contribution from the youngest stellar population is higher than when considering the whole Sgr B1 region analyzed, suggesting an over density of young stars in this region. We also found that the contribution from stars in the age bin between 0.5-2 Gyr is more important, caused by a more significant contribution from the MIST models (that is significantly higher than the Parsec models' contribution) to the final result.

To assess the results, we analyzed the variation of the contribution of the youngest stellar bin when considering potential sources of systematic uncertainties, as we did in Sect. V for the whole Sgr B1 region. We concluded that the contribution of the youngest stellar population, and thus the detection of one or several dissolved clusters, is unaffected when considering: different bin widths of the KLF, variations of the faint and bright ends of the KLF, a top-heavy IMF, and a different completeness solution. On the other hand, we measured a somewhat higher contribution of the youngest stellar bin when considering solar metallicity ( $14 \%$ of the total stellar mass is due to the youngest stellar population), and 1.5 solar metallicity ( $10 \%$ of the stellar mass due to the youngest 
stellar population). Therefore, we concluded that the detection of a significant contribution ( $\gtrsim \%$ of the stellar mass) of the youngest stellar population is robust.

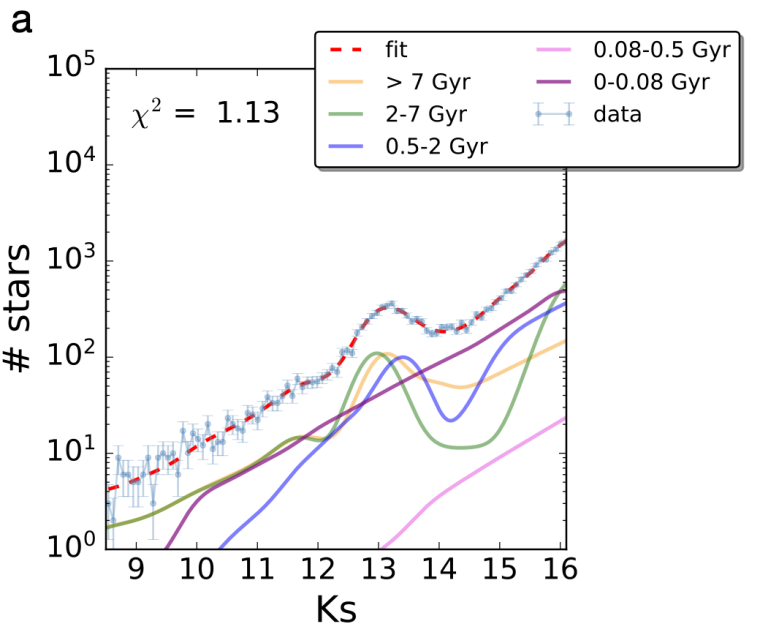

b

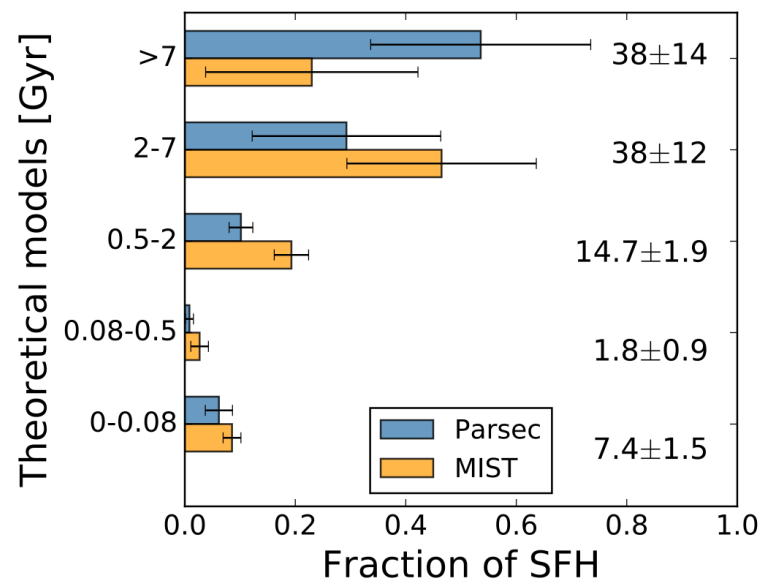

Fig. 8: a, KLF (in blue) obtained for the region of intense hot dust emission in the Spitzer $4.5 \mu$ image (Fig. 1). The error bars show the 1 ouncertainties. The red dashed line depicts the best-fit model (Parsec models). Colored lines show the contribution of the different age bins. $\boldsymbol{b}$, SFH of the region of intense hot dust emission. The error bars indicate the $1 \sigma$ uncertainties. The numbers indicate the mean fractions of originally formed stellar mass and their uncertainties.

\section{Tests with artificial SFHs}

We assessed the reliability of the KLF fitting method using synthetic SFHs created with Parsec models assuming twice solar metallicity. We built 4 different SFHs considering scenarios with and without the presence of young stars (Fig. 9). We assumed a stellar mass of $\sim 2.2 \cdot 10^{6} M_{\odot}$, similar to the one obtained when analyzing the region of intense hot dust emission (the region with lowest stellar mass and thus, the most challenging case). We simulated the uncertainties for each stellar bin in agreement with real uncertainties computing them as the square root of the number of stars in a given magnitude bin. Figure 9a shows the simulated KLFs and also how they present different relative contribution of their characteristic features that allows us to reconstruct the SFH via model fitting. We applied the same analysis as for real data, and obtained that the method is able to recover all the simulated SFHs within the $1 \sigma$ uncertainties (Fig. 9b).

a

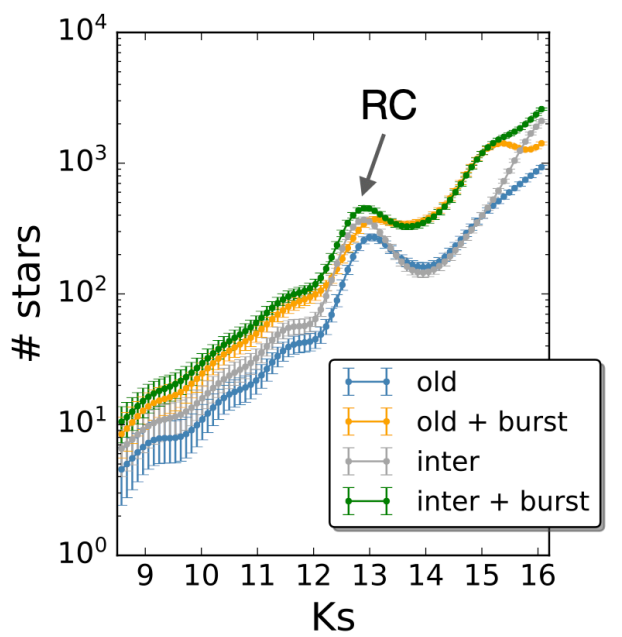

b

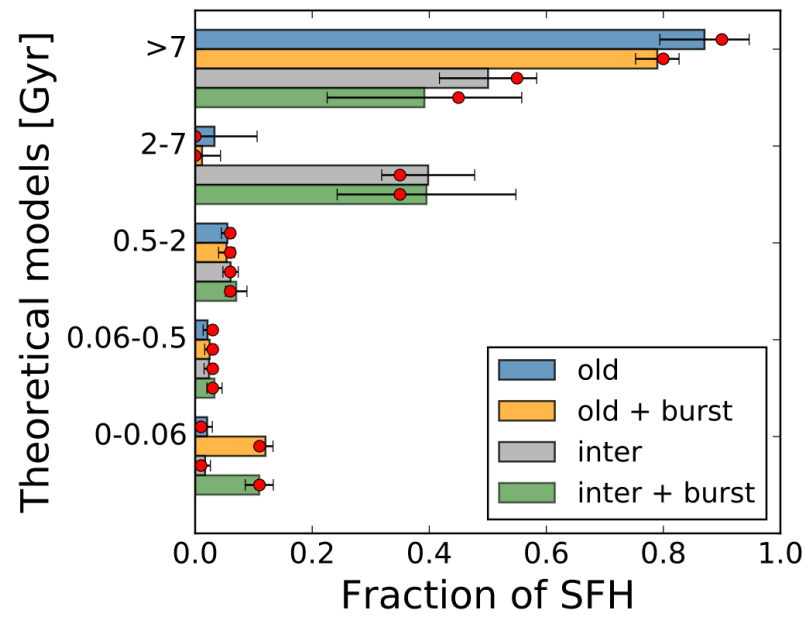

Fig. 9: a, Simulated KLFs with similar initial stellar mass corresponding to different SFHs (red dots in Fig. 8b). The error bars show the $1 \sigma$ uncertainties, that were estimated assuming Poisson errors. The 
position of the red clump (RC) feature is indicated by an arrow. $\boldsymbol{b}$, recovered SFHs after applying the model fitting technique. The error bars depict $1 \sigma$ uncertainties.

\section{Comparison with the Quintuplet cluster}

We compared the results obtained for the region of intense hot dust emission with a same size region containing the Quintuplet cluster (F10 field of the GALACTICNUCLEUS survey ${ }^{13}$, see Sect. I). Figure 10 shows the results obtained after applying our KLF fitting technique using Parsec and MIST models. We observed that the uncertainties are higher than for the case of the Sgr B1 region with intense hot dust emission. This is due to the lower data completeness in this region that limits the faint end of KLF to Ks 15 mag ( $\sim 1$ mag lower than for the Sgr B1 region). We estimated that the contribution of the very young stars considering the $5+10$ Myr stellar models (the estimated age of the Quintuplet cluster is $\sim 5 \mathrm{Myr})$ is $1.3 \pm 0.9 \%$ of the total stellar mass in the region. This means a young stellar mass of $(6.8 \pm 0.5)$ - $10^{4} M_{\odot}$, that is of the same order of magnitude that the estimated mass for the Quintuplet cluster $\left(\sim 10^{4} M_{\odot}\right)$, revealing its presence in the analyzed region and indicating that the method is capable of identifying young stars in a given field. A more precise measurement of the total young stellar mass would require deeper photometry to better constrain the faint end of the KLF.
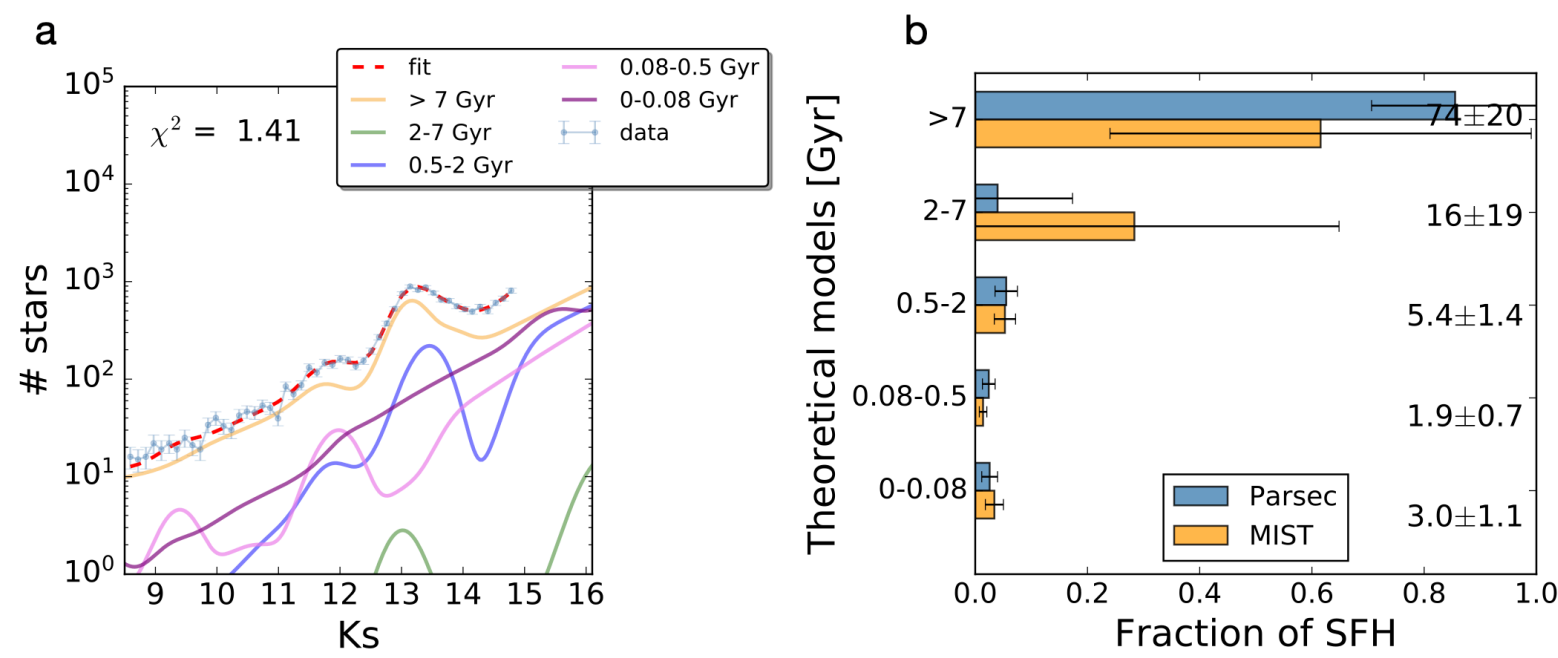

Fig. 10: a, KLF (in blue) obtained for the Quintuplet cluster region. The error bars show the $1 \sigma$ uncertainty. The red dashed line depicts the best-fit model (Parsec models), whose chi2 is indicated in the Figure. Colored lines show the contribution of the different age bins. $\boldsymbol{b}$, SFH of the Quintuplet region. The error bars indicate the $1 \sigma$ uncertainties. The numbers indicate the mean fractions of originally formed stellar mass and their uncertainties 